\title{
HIGHLY SATURATED PACKINGS AND REDUCED COVERINGS
}

\author{
GABOR FEJES TÓTH, GREG KUPERBERG, AND WŁODZIMIERZ KUPERBERG
}

\begin{abstract}
We introduce and study certain notions which might serve as substitutes for maximum density packings and minimum density coverings. A body is a compact connected set which is the closure of its interior. A packing $\mathscr{P}$ with congruent replicas of a body $K$ is $n$-saturated if no $n-1$ members of it can be replaced with $n$ replicas of $K$, and it is completely saturated if it is $n$-saturated for each $n \geq 1$. Similarly, a covering $\mathscr{C}$ with congruent replicas of a body $K$ is $n$-reduced if no $n$ members of it can be replaced by $n-1$ replicas of $K$ without uncovering a portion of the space, and it is completely reduced if it is $n$-reduced for each $n \geq 1$. We prove that every body $K$ in $d$-dimensional Euclidean or hyperbolic space admits both an $n$-saturated packing and an $n$-reduced covering with replicas of $K$. Under some assumptions on $K \subset \mathbb{E}^{d}$ (somewhat weaker than convexity), we prove the existence of completely saturated packings and completely reduced coverings, but in general, the problem of existence of completely saturated packings and completely reduced coverings remains unsolved. Also, we investigate some problems related to the the densities of $n$-saturated packings and $n$-reduced coverings. Among other things, we prove that there exists an upper bound for the density of a $d+2$ reduced covering of $\mathbb{E}^{d}$ with congruent balls, and we produce some density bounds for the $n$-saturated packings and $n$-reduced coverings of the plane with congruent circles.
\end{abstract}

\section{INTRODUCTION AND PRELIMINARIES}

Two of the basic problems in the theory of packing and covering are to determine the most efficient packing and covering with replicas of (meaning sets congruent to) a given set $K$ in some metric space. Recall that a packing is a family of sets whose interiors are mutually disjoint, and that a covering is a family of sets whose union is the whole space. By a space we mean either $d$-dimensional Euclidean space $\mathbb{E}^{d}$ or $d$-dimensional hyperbolic space $\mathbb{H}^{d}$, although the definitions that follow are sometimes more general. We shall consider packings and coverings with replicas of a nonempty compact connected set which is the closure of its interior, a body, for short.

The usual measure of the efficiency of an arrangement in Euclidean space is density. Roughly speaking, the density of an arrangement is the total volume of the members of the arrangement divided by the volume of the whole space. Rigorously, density can be defined by an appropriate limit [FK93]. The maximum density of a packing of the space with replicas of a (measurable) set $K$ is denoted by $\delta(K)$ and is called the packing density of $K$. The minimum density of a covering with replicas of $K$ is denoted by $\vartheta(K)$ and is called the covering density of $K$. It is known that each of the maximum and the minimum density is attained [Gro63].

There are some disadvantages of using density for measuring the efficiency of an arrangement. In the first place, optimum density is a global notion and it does not imply the local efficiency of an arrangement. Secondly, the notion of density cannot be extended in full generality to hyperbolic geometry [Bör74], [FK93]. In what follows we introduce and study certain notions which might serve as substitutes for maximum density packings and minimum density coverings.

Let $K$ be a body and let $\mathscr{P}$ be a packing of space with replicas of $K . \mathscr{P}$ is said to be saturated if it cannot be augmented with any additional replica of $K$ without overlapping with a member of $\mathscr{P}$. More generally, $\mathscr{P}$ is $n$-saturated if no $n-1$ members of it can be replaced with $n$ replicas of $K$. A packing is completely saturated if it is $n$-saturated for every $n \geq 1$.

Note that Fejes Tóth and Heppes [FH80] define the term " $n$-saturated" differently, but we hope that our definition causes no confusion.

Date: November 24, 1995. 
A covering $\mathscr{C}$ of $E^{d}$ with replicas of $K$ is reduced if no proper sub-family of $\mathscr{C}$ is a covering. Similarly, we say that $\mathscr{C}$ is $n$-reduced if no $n$ members of it can be replaced by $n-1$ replicas of $K$ without uncovering a portion of the space. A covering is completely reduced if it is $n$-reduced for every $n \geq 1$.

Conjecture . Every body $K$ in $\mathbb{E}^{d}$ (resp. in $\mathbb{H}^{d}$ ) admits a completely saturated packing and a completely reduced covering with replicas of $K$.

This conjecture is supported by the following results:

Theorem 1.1. Every convex body in $\mathbb{E}^{d}$ admits a completely saturated packing and a completely reduced covering of $\mathbb{E}^{d}$ with replicas of the body.

Theorem 1.2. Every body $K$ in $\mathbb{E}^{d}$ (resp. in $\mathbb{H}^{d}$ ) admits both an $n$-saturated packing and an $n$-reduced covering with replicas of $K$.

Section 2 presents a proof of Theorem 1.1. We note there that the theorem holds for bodies satisfying the strict nested similarity property, a condition weaker than convexity. Theorem 1.2 is proved in Section 3 (for the Euclidean case) and Section $\theta$ (for the hyperbolic case), each as a corollary of a more general statement. The hyperbolic case involves some elements of the theory of hyperbolic manifolds, which we review for the benefit of the unfamiliar reader.

1.1. Acknowledgements. The authors acknowledge, with gratitude, that during the preparation of this paper, the research of G. Fejes Tóth has been supported by the Hungarian Foundation for Scientific Research (OTKA), grants no. 1907 and no. 14218, and that of W. Kuperberg by the National Science Foundation, grant no. DMS-9403515.

\section{COMPLETE SATURATION AND REDUCTION}

In this section, we give a proof of Theorem 1.1. We precede the proof with some definitions and two lemmas. Throughout the argument, $K$ is a given convex body in $\mathbb{E}^{d}$, and, as before, $V(A)$ denotes the volume of $A$. We use the Hausdorff distance between closed sets to measure the distance between a pair of (finite) packings or coverings, extending the Hausdorff distance function to the space of finite (unordered) collections of compact sets in the natural way.

Let $c$ be a "center" point in the interior of $K$, say the center of gravity of $K$. Let $B(r, p)$ be the sphere of radius $r$ centered at $p$. A packing with replicas of $K$ is completely saturated in $B(r, p)$ if no $n$ replicas contained in $B(r, p)$ can be replaced by $n+1$ replicas contained in $B(r, p)$, for every integer $n$ (the replicas not contained in $B(r, p)$ are not to be moved in this process). A packing is unsaturated in $B(r, p)$ for short if it fails to be completely saturated in $B(r, p)$. An arbitrary packing of $\mathbb{E}^{d}$ can be altered within $B(r, p)$ so as to result in a packing completely saturated in the ball: delete all replicas of $K$ contained in the ball and replace them with the maximum number of replicas of $K$ that will fit in the ball without overlapping with each other or with any of the replicas that partially invade the ball.

A homothetic thinning $T_{h}(\mathscr{P})$ of a packing $\mathscr{P}$ with replicas of $K$ by a factor of $h>1$ is a new packing such that each center $c \in \mathbb{E}^{d}$ maps to $h c$, but such that the replicas of $K$ are translated without expansion. The analogous concepts for coverings (completely reduced in $B(r, p)$, unreduced in $B(r, p)$, homothetic thickening by a factor of $h<1$ ) are defined similarly. The proof of Theorem 1.1 is given only for packings, since the proof for coverings is the same except for one modification which is mentioned afterwards.

Lemma 2.1. For every $r$ and $\varepsilon>0$, there exists a $\delta>0$ such that if a $\mathscr{P}$ is less than $\delta$ away (in Hausdorff distance) from a packing which is unsaturated in $B(r, 0)$, then $T_{1+\varepsilon}(\mathscr{P})$ is unsaturated in $B((1+\varepsilon) r, 0)$.

The proof is left to the reader.

Lemma 2.2. Let $r>0$ and $\eta>0$. Then there exists an $s_{0}$ and $a \delta>0$ such that for every $s>s_{0}$, a packing $\mathscr{P}$ of replicas of $K$ which is densest relative to $B(2 s+r, 0)$ has the following property: If $p$ is chosen at random in $B(s, 0), \mathscr{P}$ is at least $\delta$ away from unsaturated in $B(r, p)$ with probability at least $1-\eta$. 
Proof. Informally, if $\varepsilon$ is sufficiently small and $s$ is sufficiently large, then if $\mathscr{P}$ is expanded by $1+\varepsilon$, the loss of density from replicas of $K$ sliding over the edge of $B(2 s R+r, 0)$ is outweighed by the gain in finding a $\eta$ proportion of $B(r, p)$ 's inside that are unsaturated and re-saturating the packing in a disjoint collection of these smaller balls. Then $\delta$ can be chosen based on $\varepsilon$ and Lemma 2.1. A more precise argument follows.

Temporarily fix $\delta>0$ and $s>0$, and suppose that, to the contrary, the set $X$ of points $p \in B(s, 0)$ such that $\mathscr{P}$ is less than $\delta$ away from unsaturated in $B(r, p)$ has measure at least $\eta V(B(s, 0))$. We will arrive at a contradiction for $\delta$ sufficiently small and $s$ sufficiently large.

Since $X$ has measure $\eta V(B(s, 0))$, it cannot be covered by fewer than

$$
\eta \frac{V(B(s, 0))}{V(B(2 r, 0))}=\frac{\eta}{(2 r)^{d}} s^{d}
$$

balls of radius $2 r$. It follows that there exists a packing $\left\{B\left(r, p_{i}\right)\right\}_{1 \leq i \leq k}$ of $k$ balls of radius $r$ entirely within $B(s+r, 0)$ such that the restriction of $\mathscr{P}$ to each ball is less than $\delta$ away from an unsaturated packing, where

$$
k>\eta \frac{V(B(s, 0))}{V(B(2 r, 0))}>c s^{d}
$$

for some constant $c$ depending only on $r$ and $\eta$. In other words,

$$
\frac{k V(K)}{V(B(s, 0))}>\frac{c V(K)}{V(B(1,0))} \text {. }
$$

Let

$$
C=\frac{c V(K)}{V(B(1,0))}<1
$$

let

$$
\varepsilon=\min \left((1-C)^{-\frac{1}{d}}-1, \frac{1}{2}\right)
$$

and let $\delta$ be given by Lemma 2.1.

Observe that the difference between the density of $\mathscr{P}$ and that of the homothetic thinning $T_{1+\varepsilon}(\mathscr{P})$ (both relative to $B(s, 0))$ is at most $1-(1-\varepsilon)^{-d}$. On the other hand, since $T_{1+\varepsilon}(\mathscr{P})$ is unsaturated in each of the balls $B\left((1+\varepsilon) r,(1+\varepsilon) p_{i}\right)$, the density of $T_{1+\varepsilon}(\mathscr{P})$ relative to $B(s, 0)$ can be increased through saturation by an amount greater than

$$
k(s) V(K) / V(B(s, 0)>c V(K) / V(B(1,0))=C .
$$

(Note that by our choice of $\varepsilon$, each $B\left((1+\varepsilon) r,(1+\varepsilon) p_{i}\right)$ is contained in $B(2 s+r, 0)$.) By our choice of $\varepsilon$, we have $1-(1-\varepsilon)^{-d}<C$. Thus, the thinned and then re-saturated packing is denser in $B(2 s+r, 0)$ than the original packing $\mathscr{P}$, which is a contradiction.

Proof of theorem. Let $n>0$ be an integer and for each $1 \leq k \leq n$, let $\delta_{k}$ and $s_{k}$ be given by the second lemma with $r=k$ and $\eta=3^{-k}$. Let $s$ be the supremum of the $s_{k}$ 's, and let $\mathscr{P}$ be a packing which is densest relative to $B(2 s+n, 0)$. Then for at least

$$
1-\left(\frac{1}{3}+\frac{1}{9}+\ldots+\frac{1}{3^{n}}\right)>1 / 2
$$

of $p \in B(s, 0), \mathscr{P}$ is $\delta_{k}$ away from unsaturated in all $B(k, p)$ 's simultaneously. After translation by $-p, \mathscr{P}$ becomes a packing $\mathscr{P}_{n}$ which is simultaneously $\delta_{k}$ away from unsaturated in each $B(k, 0)$. The sequence $\mathscr{P}_{n}$ has a subsequence which converges in the Hausdorff topology to a limit $\widetilde{P}$. The packing $\widetilde{P}$ is $\delta_{n}$ away from unsaturated in $B(n, 0)$ for every $n>0$, and it is therefore completely saturated in $\mathbb{E}^{d}$.

REMARK 1. The argument for the analogous theorem for coverings requires two minor modifications in the formulation and proof of Lemma 2.2. Firstly, instead of using a covering which extremizes density in $B(2 s+r, 0)$, we use a minimum cardinality arrangement of replicas of $K$ that covers $B(2 s+r, 0)$. In particular, an optimal covering of $B(2 s+r, 0)$ in this sense has no replicas disjoint from $B(2 s+r, 0)$. Secondly, a homothetic thickening of a covering of $B(2 s+r, 0)$ by a factor of $1-\varepsilon$ is not in general again a covering. 
To repair it, we identify a cube $\kappa \subset K$ and we cover the annular region $B(2 s+r, 0)-B((1-\varepsilon)(2 s+r), 0)$ by non-overlapping translates of $\kappa$. The resulting gain in number of replicas due to homothetic thickening is comparable to the loss of density due to homothetic thinning.

REMARK 2. The hypothesis that $K$ is convex can be weakened somewhat without any changes in the proof. It suffices that $K$ have the strict nested similarity property, which requires that, for every positive number $h<1$, the interior of $K$ contains a replica of $h K$. For example, if $K$ is strictly starlike, i.e., $K$ contains an interior point such that every ray emanating from it meets the boundary of $K$ at a single point, then $K$ has the strict nested similarity property. Another example of a body with the strict nested similarity property is an $\varepsilon$-neighborhood of a logarithmic spiral, as shown in Figure 1 .

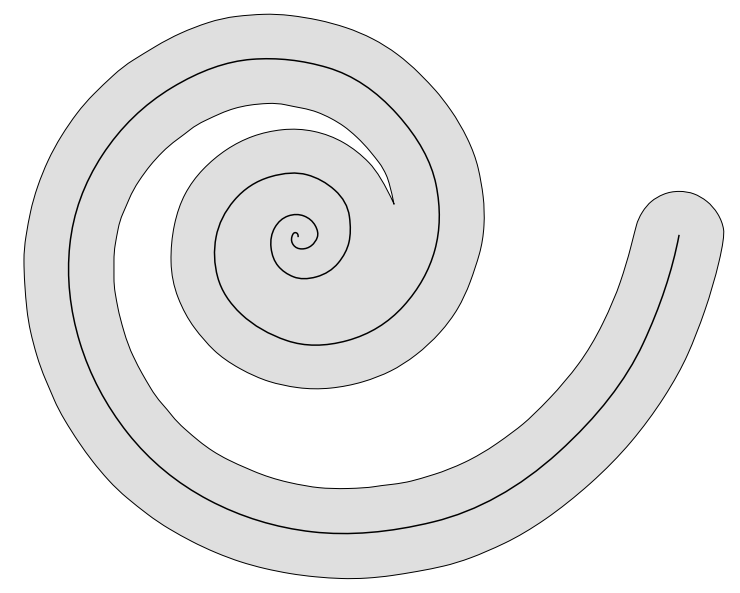

FIGURE 1. A body with the strict nested similarity property

\section{LATTICES OF ISOMETRIES}

We recall a construction from the topological theory of covering spaces, to be used in this section and in the next one, which we apply to produce $n$-saturated packings and $n$-reduced coverings in various spaces. For the basic notions and facts related to that theory we refer the reader to [Spa66, Ch. 1-2].

Let $M$ be a locally compact, connected metric space (for our purposes it is sufficient to assume that $M$ is a non-compact Riemannian manifold) with the metric $\rho$. A group $G$ of self-isometries of $M$ is a fixed-pointfree, uniform lattice of isometries, or lattice of isometries or lattice for short, if $G$ satisfies the following two conditions:

(i): there is a number $\gamma>0$ such that for every $x \in M$ and every $g \in G$ other than the identity, $\rho(x, g(x)) \geq$ $\gamma$

(ii): the quotient space $B=M / G$ (whose points are the orbits $\{g(x): g \in G\}$ of points of $M$ under $G$, furnished with the quotient topology) is compact.

Every lattice $G$ of isometries of $M$ has the crucial property that the quotient map $p$ from $M$ to $B$, assigning to each point of $M$ its orbit, is a covering projection (see [Spa66, p. 88]). We shall refer to $M$ as the covering space and to $B$ as the base space. Also, the covering is regular, for $G$ acts transitively on each point-inverse. The base space is metrizable: a specific metric for $B$ is defined by $\rho(x, y)=\inf \{\rho(\tilde{x}, \tilde{y}): p(\tilde{x})=x, p(\tilde{y})=y\}$. Under this metric, the covering projection $p$ is a local isometry: the restriction of $p$ to any set of diameter smaller than $\gamma$ is an isometry.

Conversely, given a regular covering $p: M \rightarrow B$, where $B$ is a compact connected manifold, $M$ is connected and endowed with the metric lifted from $B$, then the group of covering transformations of $M$ is a lattice of isometries. Moreover, if $M$ is simply connected, then the lattice of isometries of $M$ is isomorphic to the fundamental group $\pi_{1}(B)$ of the base space $B$ (see [Spa66, Sec. 2.6]). 
If $M$ is $d$-dimensional Euclidean space, then a lattice of isometries which consists of translations reduces to the classical concept of a lattice of vectors, and the resulting base space is a $d$-dimensional torus. However, there are other lattices of isometries even in $\mathbb{E}^{2}$. For instance, the group of isometries of the Cartesian plane generated by a translation in the $x$ direction and a glide-reflection in the $y$ direction is a lattice of isometries, but the resulting base space is a Klein bottle and not a torus.

Define the girth of a lattice $G$ as the infimum of the distances $\rho(x, g(x))$ over all non-identity elements $g \in$ $G$. If $M$ is a simply-connected Riemannian manifold (such as $\mathbb{H}^{d}$ ), then the same number is the infimum of the lengths of all non-trivial loops in the base space, hence the name "girth". For many manifolds, including Euclidean and hyperbolic manifolds, the girth is twice the injectivity radius of the quotient manifold, which is defined as the largest $r$ such that no metric ball of radius $r$ overlaps itself.

Observe that if $S$ is a subset of $M$ whose diameter is smaller than the girth of $G$, then the image $p(S)$ is a replica of $S$ in $B$. Also, the set $p^{-1}(p(S))$ is the union of a discrete collection of mutually disjoint replicas of $S$, namely it is the orbit of $S$ under $G$. We call this discrete collection of replicas of $S$ a lifting of $p(S)$ (in $M)$.

Theorem 3.1. Let $M$ be a locally compact connected metric space and let $K$ be a body in $M$. If for every $c>0, M$ admits a lattice of girth greater than $c$, then there exist an n-saturated packing of $M$ and an $n$-reduced covering of $M$ with replicas of $K$, for every positive integer $n$.

Proof. We restrict our attention to packings only, since the case of coverings is completely analogous.

Let $G$ be a lattice of isometries of $M$ of girth greater than $2 n+1$ times the diameter of $K$, let $B$ be the base space associated with the lattice, and let $p: M \rightarrow B$ be the covering projection. In the base space $B$, arrange a packing with a maximum number of bodies of the form $p\left(K^{\prime}\right)$, where $K^{\prime}$ is a replica of $K$ in $M$. The maximum is finite because $B$ is compact and $K$ has a non-empty interior. Let $\mathscr{P}$ be the lifting (in $M$ ) of this packing. Obviously, $\mathscr{P}$ is a packing of $M$ with replicas of $K$. We assert that $\mathscr{P}$ is $n$-saturated.

Suppose the contrary, and let $m \leq n$ be the smallest positive integer such that $\mathscr{P}$ is not $m$-saturated. Therefore there are $m$ members of $\mathscr{P}$, say $K_{1}, K_{2}, \ldots, K_{m}$ which can be replaced by $m+1$ other replicas of $K$, say $L_{1}, L_{2}, \ldots, L_{m+1}$, and $m$ is the smallest integer with this property. By the "pigeonhole principle," the set $S=\left(\cup K_{i}\right) \cup\left(\cup L_{j}\right)$ is connected. Therefore the diameter of $S$ is smaller than the sum of the diameters of the $K_{i}$ 's and the $L_{j}$ 's, thus smaller than the girth of $G$. If we replace in $B$ the sets $p\left(K_{i}\right)(i=1,2, \ldots, m)$ with the sets $p\left(L_{j}\right)(j=1,2, \ldots, m+1)$, we exceed the maximum number defined above. This is a contradiction.

The Euclidean case of Theorem 1.2 is an immediate corollary of Theorem 3.1. Also, the remaining part of Theorem 1.2 is now reduced to the problem of existence of lattices of arbitrarily large girth in $d$-dimensional hyperbolic space. This problem is addressed in the next section.

\section{Hyperbolic LATTICES OF LARGE GIRTH}

A lattice of isometries of hyperbolic space $\mathbb{H}^{d}$ will be called a ( $d$-dimensional) hyperbolic lattice for short. The aim of this section is to prove the following:

Theorem 4.1. For every $c$, there exists a d-dimensional hyperbolic lattice of girth greater than $c$.

Although this fact and the methods used for proving it have been known for a long time, we could not find a suitable reference and we include a proof for completeness.

We begin with some algebraic preliminaries. A group $G$ is residually finite if for every $g \in G$ other than the identity $e$, there exists a normal subgroup $N$ of finite index which does not contain $g$. Equivalently, $G$ is residually finite if for every $g \in G, g \neq e$, there is a homomorphism $\varphi$ from $G$ to a finite group such that $\varphi(g)$ is not the identity.

Since the intersection of two normal, finite-index subgroups of $G$ is again a normal subgroup of finite index, we get immediately:

Proposition 4.2. If $G$ is residually finite, then for any finite set $F \subset G$ not containing the identity there exists a normal subgroup $N \subset G$ of finite index which does not intersect $F$. 
The group of non-singular $n \times n$ matrices with real coefficients is denoted by $G L(n, \mathbb{R})$ and $I\left(\mathbb{H}^{d}\right)$ denotes the group of isometries of $\mathbb{H}^{d}$. Since $\mathbb{H}^{d}$ can be modelled as one sheet of a two-sheeted hyperboloid in $\mathbb{R}^{d+1}$, where the isometries of $\mathbb{H}^{d}$ are those linear transformations of $\mathbb{R}^{d+1}$ which preserve the sheet (see [BP92, Sec. A2]), the group $I\left(\mathbb{H}^{d}\right)$ is isomorphic to a subgroup of $G L(d+1, \mathbb{R})$.

The following lemma is a direct consequence of a theorem of Mal'cev ([ Mal40, Th. VII $]$ ). We include a version of Mal'cev's proof.

Lemma 4.3. Every finitely generated subgroup $G$ of $G L(n, \mathbb{R})$ is residually finite.

Proof. Let $g$ be a non-identity element of $G$. The aim of the proof is to construct a homomorphism from $G$ to a finite group such that the image of $g$ is also not the identity. This is accomplished by the composition of three homomorphisms. The first one, $\alpha$, sends $G$ into the group of algebraic matrices (matrices whose entries are algebraic numbers) of the same size as the matrices in $G$; the second one, $\beta$, is a map to a group of (larger) rational matrices; and the third one, $\gamma$, is a map to a group of matrices over a finite field $\mathbb{Z} / p$.

Let $g_{1}, g_{2}, \ldots, g_{k}$ be a set of generators for $G$. If a set of algebraic equations in finitely many variables has a real solution, then it has (possibly complex) algebraic solutions arbitrarily close to the real solution. The defining relations between the $g_{i}$ 's impose some constraints on the entries of these matrices. Since the constraints are algebraic, algebraic matrices can be found that satisfy the same relations as the $g_{i}$ 's do, and are arbitrarily close to them. We pick algebraic matrices for the images under $\alpha$ of the generators that are close enough to the original real matrices so that $g$ 's image $\alpha(g)$ is not the identity.

The coefficients of all of the $\alpha\left(g_{i}\right)$ 's are algebraic numbers that, all together, lie in some field $F$ which is a finite-dimensional vector space over $\mathbb{Q}$. The algebraic numbers can then themselves be understood as rational linear transformations of $F$. Therefore, possibly by passing to larger matrices, we can assign to each matrix $\alpha\left(g_{i}\right)$ a larger matrix with rational entries, and this assignment extends to a monomorphism $\beta$. Thus, $\beta \alpha(h)$ is a rational matrix assigned to $h$ for every $h \in G$.

To define the third and last homomorphism, let $p$ be a prime which does not divide the denominator of any $\beta \alpha\left(g_{i}\right)$. (The prime $p$ therefore also does not divide the denominator of any coefficient of any $\beta \alpha(h)$.) In general, if $p$ does not divide $b$, the fraction $a / b$ is well-defined as an element of $\mathbb{Z} / p$. Therefore we can reduce all $\beta \alpha\left(g_{i}\right)$ 's mod $p$ to obtain a homomorphism $\gamma$ of $\beta \alpha(G)$ to a group of matrices over $\mathbb{Z} / p$ if $p$ fails to divide all denominators in all $\beta \alpha\left(g_{i}\right)^{\prime}$ s. We know that $\beta \alpha(g)$ for the originally-chosen $g$ is not the identity matrix. Therefore if $p$ is larger than all numerators in the matrix $\beta \alpha(g)$ as well, then $\gamma \beta \alpha(g)$ will also be distinct from the identity.

Corollary 4.4. Every hyperbolic lattice is residually finite.

Proof. For a $d$-dimensional hyperbolic lattice $G$, the base space $B=\mathbb{H}^{d} / G$ is a smooth and closed, hence triangulable, manifold. Therefore the fundamental group $\pi_{1}(B)$ is finitely generated. In effect, $G$ is isomorphic to a finitely generated group of matrices.

Proof of theorem. The proof consists of two parts. In the first part we show the existence of a $d$-dimensional hyperbolic lattice, and in the second part, given a number $c$, we show that every hyperbolic lattice contains a sublattice $G^{\prime}$ whose girth is greater than $c$. For the first part, we quote directly from the introduction to [Bor63, p. 111]:

A Clifford-Klein form of a connected and simply connected Riemannian manifold $M$ is a Riemannian manifold $M^{\prime}$ whose universal Riemannian covering (universal covering endowed with the metric lifted from $M^{\prime}$ ) is isomorphic to $M$. The main purpose of this note is to prove the following:

Theorem A. A simply connected Riemannian symmetric space $M$ always has a compact Clifford-Klein form. Any such form $M^{\prime}$ has a finite Galois covering which is proper, unless $M^{\prime}$ is isomorphic to $M$.

We recall that a Riemannian manifold $X$ is symmetric, in the sense of Cartan, if it is connected and if every point $x$ in $X$ is an isolated fixed point of an involutive isometry $s_{x}$. 
Obviously, hyperbolic space $\mathbb{H}^{d}$ is a simply-connected Riemannian symmetric space, and a compact Clifford-Klein form of $\mathbb{H}^{d}$ produces immediately a lattice of isometries of $\mathbb{H}^{d}$ as the group of covering transformations.

For the second part of the proof, assume that $G$ is a lattice of isometries of $\mathbb{H}^{d}$ and let $c>0$. Let $B$ be the quotient manifold $\mathbb{H}^{d} / G$ and let $p: \mathbb{H}^{d} \rightarrow B$ be the covering map. The girth of $G$ is determined by the shortest non-contractible (unbased) loop in $B$.

If $\lambda$ is a non-contractible unbased loop, $\lambda$ represents a conjugacy class of elements of $\pi_{1}(M)$. If $g$ is an element of this conjugacy class and $N$ is a normal subgroup of $\pi_{1}(M)$ that does not contain $g$, then $N$ does not contain any conjugate of $g$ either. Since $B$ is compact, it admits only finitely many homotopy classes with loops of length at most $c$, say $\left\{\left[\lambda_{1}\right],\left[\lambda_{2}\right], \ldots,\left[\lambda_{k}\right]\right\}$. Each $\left[\lambda_{i}\right]$ represents a conjugacy class of an element $g_{i} \in \pi_{1}(M)$. Let $F=\left\{g_{1}, g_{2}, \ldots, g_{k}\right\}$. By Corollary 4.4 and Proposition 4.2, $\pi_{1}(M)$ has a finite-index normal subgroup $N$ that does not intersect $F$. Thus $N$ does not contain any conjugate of any of the $g_{i}$ 's.

Let $q: \widetilde{B} \rightarrow B$ be the covering of $B$ corresponding to $N$. Since $B$ is compact and $N$ has finite index, $\widetilde{B}$ is compact as well. By the universality of the covering $p: \mathbb{H}^{d} \rightarrow B$, there exists a covering $\widetilde{p}: \mathbb{H}^{d} \rightarrow \widetilde{B}$, hence $\widetilde{B}$ determines a $d$-dimensional hyperbolic lattice $\widetilde{G}$. It is clear that the girth of $\widetilde{G}$ is greater than $c$, or, in other words, the length of every non-contractible (unbased) loop in $\widetilde{B}$ is greater than $c$, because $q_{*}: \pi_{1}(\widetilde{B}) \rightarrow \pi_{1}(B)$ is a monomorphism, $q_{*} \pi_{1}(\widetilde{B})=N$ and $q$ does not increase the length of any loop.

\section{DENSE $n$-REDUCED COVERINGS}

We begin with an example of an arbitrarily dense 2-saturated lattice covering of $\mathbb{E}^{d}$ with unit balls $(d \geq 2)$. Let $e_{1}, e_{2}, \ldots, e_{d}$ be an orthonormal basis for $\mathbb{E}^{d}$. Consider the lattice generated by the vectors $v_{i}=a e_{i}$ for $1 \leq i \leq d-1$ and

$$
v_{d}=\left(1+\sqrt{1-\frac{a^{2}}{4}(d-1)}\right) e_{d}+\frac{a}{2} \sum_{i=1}^{d-1} e_{i},
$$

where $0<a<\frac{2}{\sqrt{d-1}}$.

Clearly, the unit balls centered at the lattice points form a (simply-)reduced covering. Moreover, each ball covers pairs of points not contained in any other ball such that the distance between them approaches 2. Therefore, if two balls are deleted, then one can find four uncovered points that form the vertices of a parallelogram with two sides of lengths approaching 2 . Since no such set of four points can be covered by a single unit ball, the covering is 2-reduced. However, the density of the covering is arbitrarily large for sufficiently small $a$. Figure 2 illustrates this covering for $d=2$.

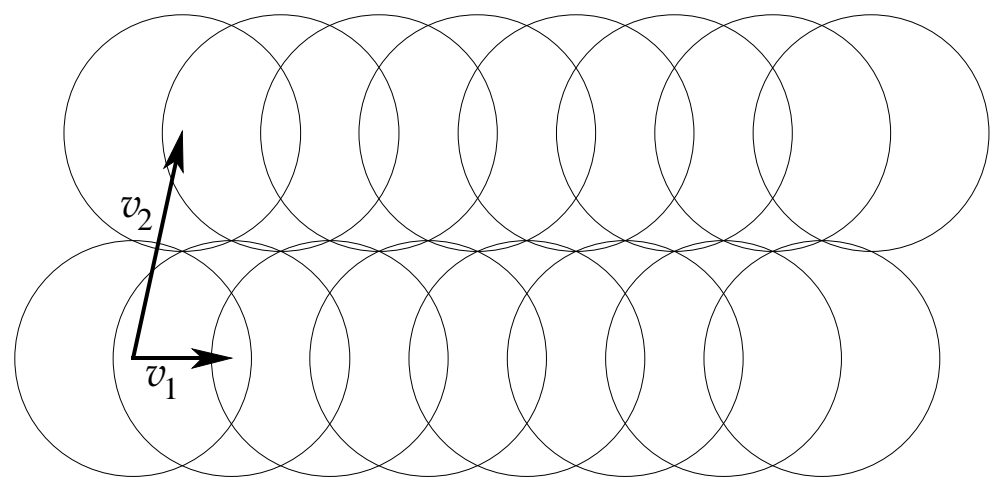

FIGURE 2. A high-density, 2-reduced covering by circles.

An equally simple construction yields an infinitely dense 2-saturated covering of $\mathbb{E}^{d}$ with unit balls ( $d \geq$ 2 ), in fact locally infinitely dense at every point. Let $P$ be a hyperplane in $\mathbb{E}^{d}$ containing the origin and let $Q$ 
be a dense subset of $P$ such that $P \backslash Q$ is dense in $P$ as well. Let $v$ denote the vector normal to $P$ of length 2. The collection of unit balls centered at the points of the form $q+2 i v$ for $q \in Q$ and $p+(2 j+1) v$ for $p \in P \backslash Q, i, j \in \mathbb{Z}$, is a covering. Since the removal of any single ball from this collection uncovers both end points of the the ball's diameter parallel to $v$, the covering is not only reduced, but 2-reduced as well.

The second construction generalizes to an arbitrary body by choosing the hyperplane $P$ to be perpendicular to a diameter of the body and giving $v$ the same length as the diameter.

Let $\Theta_{n}(K)$ be the supremum of the densities of all $n$-reduced coverings with $K$. For example, the above constructions show that $\Theta_{1}(K)=\Theta_{2}(K)=\infty$. However, the simple relation

$$
\lim _{n \rightarrow \infty} \Theta_{n}(K)=\vartheta(K)
$$

implies the existence of a smallest positive integer $l(K)$ such that $\Theta_{n}(K)<\infty$ for all $n \geq l(K)$. The notion of the Newton covering number of a convex body (introduced below) yields an upper bound for $l(K)$, where $K$ is an arbitrary convex body in $\mathbb{E}^{d}$. In addition, an application of a theorem of Bárány yields a slightly better bound for $l\left(B^{d}\right)$, where $B^{d}$ denotes the unit ball in $\mathbb{E}^{d}$.

Recall that the Hadwiger covering number $H_{c}(K)$ of a convex body $K$ in $\mathbb{E}^{d}$ is the minimum number of translates of $K$ whose union contains a neighborhood of $K$. Hadwiger [Had57] asks for the maximum value of $H_{c}(K)$ over all convex bodies $K$ in $\mathbb{E}^{d}$. The problem was stated independently by others, also in the context of the equivalent problem of illumination of $K$, and it is conjectured that $H_{c}(K) \leq 2^{d}$ with equality for parallelotopes only. The conjecture is still open in every dimension $d \geq 3$.

Similarly, one can consider $N_{c}(K)$, the minimum number of replicas of $K$ whose union contains a neighborhood of $K$. The quantities $N_{c}(K)$ and $H_{c}(K)$ have their dual counterparts $N(K)$ and $H(K)$, called the Newton (or kissing) number and the Hadwiger number, respectively, in the context of packings (see [FK93]). We call $N_{c}(K)$ the Newton covering number of $K$ to extend the analogy. Obviously, $N_{c}(K) \leq H_{c}(K)$ for all $K$.

The following theorem establishes a relation between $l(K)$ and $N_{c}(K)$.

Theorem 5.1. For every convex body $K$ in Euclidean space,

$$
l(K) \leq N_{c}(K)+1
$$

In the proof of this theorem, as well as in the next section, we use certain relations between the (global) density of an arrangement and its density with respect to some bounded domain. In what follows, the volume of a (measurable) set $S$ will be denoted by $V(S)$. As usual, $B^{d}$ denotes the unit ball in $\mathbb{E}^{d}$, so $r B^{d}$ is the ball of radius $r$ centered at the origin. Let $\mathscr{A}$ be a locally finite arrangement of uniformly bounded measurable sets, and let $G$ be a bounded domain. The density $d(\mathscr{A} \mid G)$ of $\mathscr{A}$ relative to $G$ is defined by

$$
d(\mathscr{A} \mid G)=\frac{1}{V(G)} \sum_{A \in \mathscr{A}} V(A \cap G)
$$

and the average density $d_{a v}(\mathscr{A} \mid G)$ of $\mathscr{A}$ relative to all translates of $G$ is defined by

$$
d_{a v}(\mathscr{A} \mid G)=\lim _{r \rightarrow \infty} \frac{1}{V\left(r B^{d}\right)} \int_{r B^{d}} d(\mathscr{A} \mid(G+x)),
$$

provided the limit exists. Otherwise we take the lim sup or the lim inf, and it is usually clear from context which limit is meant. Also, the domain of the integral above is $r B^{d}$, just as $r B^{d}$ is frequently used to define the density of $\mathscr{A}$ as a limit.

The following proposition is derived from these definitions by routine methods of real analysis, interchanging sums and limits with integrals, and applying Fubini's theorem.

Proposition 5.2. For every locally finite arrangement $\mathscr{A}$ of uniformly bounded measurable sets, and any bounded domain $G$, the average density $d_{a v}(\mathscr{A} \mid G)$ coincides with the density of $\mathscr{A}$.

As a direct corollary, we get: 
Proposition 5.3. Let $\mathscr{A}$ be a locally finite arrangement of uniformly bounded measurable sets, and let $G$ be a bounded domain. If the density of $\mathscr{A}$ is a, then there exists a translate of $G$ such that $d(\mathscr{A} \mid G) \geq a$ and there exists a translate of $G$ such that $d(\mathscr{A} \mid G) \leq a$.

Proposition 5.2 can be put in an equivalent, discrete form:

Proposition 5.4. Let $D$ be a locally finite set of points, and let $G$ be a bounded domain. If the number density of $D$ is $a$, then the average number of points contained in a translate of $G$ is equal to aV $(G)$.

Again, as a corollary, we get:

Proposition 5.5. Let $D$ be a locally finite set of points, and let $G$ be a bounded domain. If the number density of $D$ is $a$, then there exists a translate of $G$ which contains at least aV $(G)$ points of $D$ and there exists a translate of $G$ which contains at most $a V(G)$ points of $D$.

Proof of theorem. By definition, there exists an $\varepsilon>0$ such that some $N_{c}(K)$ replicas of $K$ cover the $\varepsilon$ neighborhood (the outer parallel domain of radius $\varepsilon$ ) of $K$. Let $p \in K$, and for each member $K_{i}$ of the covering, let $p_{i}$ be the image of $p$ under an isometry that takes $K$ to $K_{i}$. Since the group of isometries of space that fix $p$ is compact, it can be partitioned into a finite collection of sets such that, if $g$ and $h$ belong to the same set, then the Hausdorff distance between $g(K)$ and $h(K)$ is smaller than $\varepsilon / 2$, i.e., each of $g(K)$ and $h(K)$ lies in the other's $\varepsilon / 2$-neighborhood.

If the covering is sufficiently dense, then by Proposition 5.5, there exists a ball of radius $\varepsilon / 2$ containing $N_{c}(K)+1$ points $p_{i}$ such that the Hausdorff distance between each two of the sets $K_{i}-p_{i}$ (each set $K_{i}$ shifted so that $p_{i}$ is moved back to the origin) is smaller than $\varepsilon / 2$. We have now $N_{c}(K)+1$ replicas of $K$, say $K_{1}, K_{2}, \ldots, K_{N_{c}(K)+1}$, such that $K_{i}$ lies in the $\varepsilon$-neighborhood of $K_{1}$ for $2 \leq i \leq N_{c}(K)+1$. By the definition of the Newton covering number of $K$, these $N_{c}(K)+1$ replicas can be replaced by $N_{c}(K)$ others without uncovering any points. Thus, every sufficiently dense covering with replicas of $K$ fails to be $\left(N_{c}(K)+1\right)$ reduced.

Since $N_{c}\left(B^{d}\right)=d+1$, the above theorem implies immediately that $l\left(B^{d}\right) \leq d+2$. However, using a result of Bárány [Bár87, Th. 2] which generalizes a theorem of Erdős and Szekeres [ES61], one can improve this inequality as follows.

Theorem 5.6. $l\left(B^{d}\right) \leq d+1$.

Proof. Bárány's theorem states: For any $\varepsilon>0$ and $d \geq 2$ there exists a constant $n(d, \varepsilon)$ such that every finite set $V \subset \mathbb{E}^{d}$ contains a subset $W \subset V,|W| \leq n(d, \varepsilon)$ with the property that for $v \in V \backslash W$ there are points $w_{1}, w_{2} \in W$ with $\measuredangle\left(w_{1} v w_{2}\right)>\pi-\varepsilon$. Given positive numbers $\varepsilon$ and $\delta<1$, if a covering of $\mathbb{E}^{d}$ with unit balls is sufficiently dense, then, by Proposition 5.5 , some ball of radius $\delta$ contains at least $(d+1)\left(\begin{array}{c}n(d, \varepsilon) \\ 2\end{array}\right)$ centers of the unit balls. If we let $V$ be the set of these centers and we apply Bárány's theorem, we obtain $d+3$ distinct points $w_{1}, w_{2}, v_{1}, v_{2}, \ldots, v_{d+1}$ in $V$ such that $\measuredangle\left(w_{1} v_{i} w_{2}\right)>\pi-\varepsilon$ for $i=1,2, \ldots, d+1$. It follows that the points $v_{1}, v_{2}, \ldots, v_{d+1}$ lie in the "double cone" $C$ (the union of two congruent non-overlapping right cones with a common base) whose apexes are $w_{1}$ and $w_{2}$ and whose angle at each apex is $2 \varepsilon$.

Let $B(p)$ be the unit ball centered at the point $p$. Each of the $d+1$ balls $B\left(v_{i}\right)(1 \leq i \leq d+1)$ is contained in the outer parallel domain $P$ of radius 1 of $C$. Observe that the set $P \backslash\left(B\left(w_{1}\right) \cup B\left(w_{2}\right)\right)$ is a neighborhood of the $d$-2-dimensional unit sphere centered at the midpoint of $w_{1} w_{2}$ which lies in the hyperplane perpendicular to $w_{1} w_{2}$, and that his neighborhood is arbitrarily close to the sphere for sufficiently small $\varepsilon$ and $\delta$. Since such a neighborhood can be covered by $d$ unit balls, it follows that a very dense covering of $\mathbb{E}^{d}$ with unit balls cannot be $(d+1)$-reduced. Figure 3 shows the set $P \backslash\left(B\left(w_{1}\right) \cup B\left(w_{2}\right)\right)$ in dimension 2, where $\varepsilon=\pi / 6$ and $\delta=1$ are small enough for our purpose.

Remark. The proof does not use the fact that the given collection of balls is a covering. Define an $n$-reduced arrangement (not necessarily a covering) of replicas of $K$ by the property that it is not possible to delete $n$ members of the arrangement and replace them with some $n-1$ replicas of $K$ without uncovering any point 


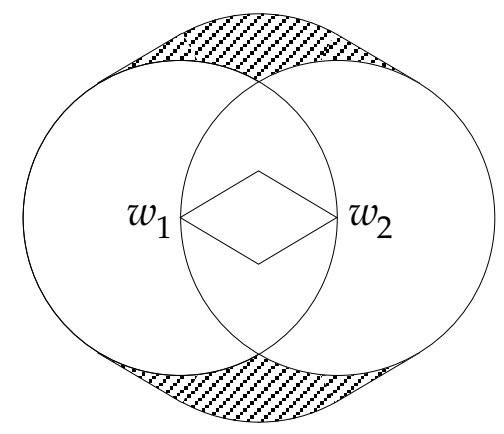

FIGURE 3. The set $P \backslash\left(B\left(w_{1}\right) \cup B\left(w_{2}\right)\right)$

that was covered by the original arrangement. Then the above argument demonstrates that a $d+1$-reduced arrangement of unit balls cannot have arbitrarily high density, and for this generalization the bound of $d+1$ is the best possible.

So far we have considered arrangements of replicas of a given body, without restrictions on the isometries that send one of them onto another. However, we can also consider arrangements with restrictions on the allowed isometries. For example, many of the ideas and results of this section and previous sections generalize to arrangements of translates of $K$ (which is only an appropriate restriction in the Euclidean case, of course). In particular, Theorem 1.2 becomes:

Theorem 5.7. Every body $K$ in $\mathbb{E}^{d}$ admits both an n-saturated packing and an $n$-reduced covering in the class of packings and coverings with translates of $K$.

Theorem 5.1 also has a "translative" version with a completely analogous proof. (In the corresponding notation, we indicate the restriction to translates by the subscript " $T$ ".)

Theorem 5.8. For every convex body $K$ in Euclidean space,

$$
l_{T}(K) \leq H_{c}(K)+1 .
$$

EXAMPLE. While the bounds given in Theorem 5.1 and Theorem 5.6 could be far from optimal, the following example indicates that Theorem 5.8 is close to optimal in at least some cases.

Let $P$ denote a right pyramid in the coordinate space $\mathbb{E}^{d}$ whose base is the unit $d$-1-dimensional cube $Q^{d-1}=\left\{\left(x_{1}, x_{2}, \ldots, x_{d-1}, 0\right) \in \mathbb{E}^{d}:\left|x_{i}\right| \leq 1 / 2\right.$ for $\left.i<d\right\}$. Observe that $H_{c}(P)=1+H_{c}\left(Q^{d-1}\right)=1+2^{d-1}$. Consider the set $\Lambda$ of vectors of the form $\left(n_{1}, n_{2}, \ldots, n_{d-1}, x\right)$ where each $n_{i}$ is an integer and $x \in \mathbb{R}$ is rational if and only if $\sum n_{i}$ is even. The translates of $P$ by all vectors of $\Lambda$ is a covering of $\mathbb{E}^{d}$ of infinite density. It is easily verified that this covering is $\left(H_{c}(P)-1\right)$-reduced. Therefore $l_{T}(P) \geq H_{c}(P)$.

The following estimate for the Hadwiger covering number of a convex body in $\mathbb{E}^{d}$ is due to Rogers (unpublished):

$$
H_{c}(K) \leq \frac{V(K-K)}{V(K)}(d \log d+d \log \log d+5 d),
$$

where $K-K$ is the difference body of $K$, consisting of points of the form $x-y$ where $x, y \in K$. The inequality follows from the result of Rogers [Rog57] which states that each $d$-dimensional convex body $K$ admits a covering of $\mathbb{E}^{d}$ by its translates of density at most $d \log d+d \log \log d+5 d$. If $\left\{K+a_{i}\right\}$ is a covering of density guaranteed by the theorem of Rogers, then Proposition 5.5 implies that there exists a $\lambda>1$ and a translate $(\lambda K)-K+c$ of $(\lambda K)-K$ containing at most $(d \log d+d \log \log d+5 d) V(K-K) / V(K)$ of the points $a_{i}$. Since $\left(K+a_{i}\right) \cap \lambda K+c \neq \varnothing$ if and only if $a_{i} \in \lambda K-K+c$, it follows that the respective translates of $K$ cover $\lambda K+c$. 
For a centrally symmetric body $K$ in $\mathbb{E}^{d}$, we have $V(K-K) / V(K)=2^{d}$, thus in this case, the Rogers bound for $H_{c}(K)$ is reasonably close to the conjectured best upper bound of $2^{d}$. In the general case, a result of Rogers and Shephard RS57] states that $V(K-K) / V(K) \leq\left(\begin{array}{c}2 d \\ d\end{array}\right)$ for every convex body $K \subset \mathbb{E}^{d}$, which yields the asymptotic bound $H_{c}(K) \leq 4^{d+o(d)}$.

\section{ASYMPTOTIC DENSITY BOUNDS}

In Section 5 , we defined $\Theta_{n}(K)$ as the supremal density of all $n$-reduced coverings with replicas of $K$, and we mentioned the simple relation

$$
\lim _{n \rightarrow \infty} \Theta_{n}(K)=\vartheta(K)
$$

Analogously, let $\Delta_{n}(K)$ be the infimum of the densities of all $n$-saturated packings with replicas of $K$, and note the analogous relation

$$
\lim _{n \rightarrow \infty} \Delta_{n}(K)=\delta(K)
$$

Also, observe that $\Delta_{n}(K)>0$ for every body $K$ and every $n \geq 1$. Obviously, each of the two sequences $\left\{\Delta_{n}(K)\right\}$ and $\left\{\Theta_{n}(K)\right\}$ is monotonic. The following inequalities give estimates for the rate of convergence of the sequences $\left\{\Delta_{n}(K)\right\}$ and $\left\{\Theta_{n}(K)\right\}$ :

$$
\Delta_{n}(K) \geq \delta(K)-O\left(n^{-1 / d}\right)
$$

and

$$
\Theta_{n}(K) \leq \vartheta(K)+O\left(n^{-1 / d}\right) .
$$

To prove inequality (6.1), assume that $K$ is a body of diameter 1 and volume $V$ and let $r$ denote the minimum radius of a ball that can intersect $n$ non-overlapping replicas of $K$. Let $\sigma_{d}$ denote the volume of the unit ball in $\mathbb{E}^{d}$. By the definition of the packing density of $K$ and Proposition 5.3,

$$
n V /\left(\sigma_{d} r^{d}\right) \geq \delta(K)-\varepsilon
$$

for every $\varepsilon>0$, hence

$$
\frac{n V}{\sigma_{d} r^{d}} \geq \delta(K)
$$

Assume now that $\mathscr{P}$ is an $n$-saturated packing with replicas of $K$, and let $p$ denote the density of this packing. Any ball of radius $r+2$ must contain at least $n$ members of $\mathscr{P}$, for otherwise the members of $\mathscr{P}$ contained in the ball could be replaced by $n$ non-overlapping replicas of $K$ intersecting the concentric ball of radius $r$. Thus the total volume of the intersections of such a ball with all members of $\mathscr{P}$ is at least $n V$. Using Proposition 5.3 again, we obtain

It follows immediately that

$$
p \geq \frac{n V}{\sigma_{d}(r+2)^{d}}
$$

$$
p \geq \delta(K)\left(\frac{r}{r+2}\right)^{d}
$$

By the definition of $r$, a ball of radius $r$ intersects at least $n$ non-overlapping replicas of $K$. Each of these replicas is contained in the concentric ball of radius $r+1$. Thus $\sigma_{d}(r+1)^{d} \geq n V$, and we get

$$
r \geq\left(\frac{V}{\sigma_{d}} n\right)^{1 / d}-1 .
$$

Since the function $f(x)=(x /(x+2))^{d}$ is increasing, we get:

$$
p \geq \delta(K)\left(\frac{c n^{1 / d}-1}{c n^{1 / d}+1}\right)^{d},
$$

where $c=\left(V / \sigma_{d}\right)^{1 / d}$, and inequality (6.1) follows. 
The proof of inequality (6.2) is analogous.

The above method can be refined as follows to yield some specific density bounds for $n$-saturated packings and $n$-reduced coverings of $\mathbb{E}^{d}$ with unit balls. For packings, consider a "cluster" of $n$ non-overlapping unit balls and let $G$ be the outer parallel domain of radius 1 of their union. Let $\mathscr{P}$ be an $n$-saturated packing with unit balls. Then every translate of $G$ contains at least $n$ centers of the members of $\mathscr{P}$. It follows, by Proposition 5.5, that the density of $\mathscr{P}$ is at least $n \sigma_{d} / V(G)$.

The smaller the volume of $G$, the greater the resulting bound, which raises the problem of arranging $n$ non-overlapping unit balls in $\mathbb{E}^{d}$ so that the volume of the outer parallel domain of radius 1 of their union is minimum. A similar method can be used for coverings, and it leads to the problem of arranging $n$ unit balls in $\mathbb{E}^{d}$, this time allowing overlaps, so that the volume of the inner parallel domain of radius 1 of their union is maximum. Of course, this method only works if $n \geq d+1$.

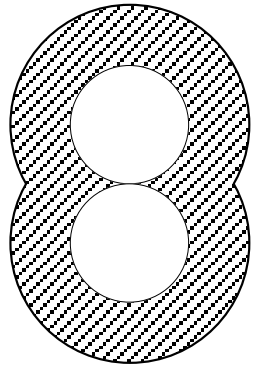

(6.3)

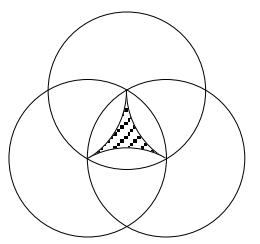

(6.6)

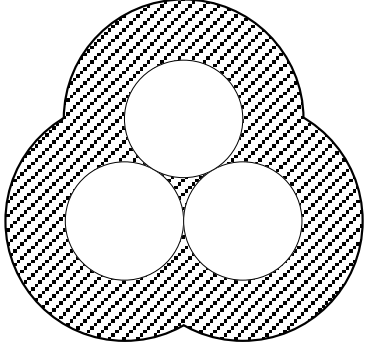

(6.4)

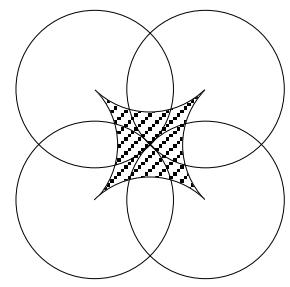

(6.7)

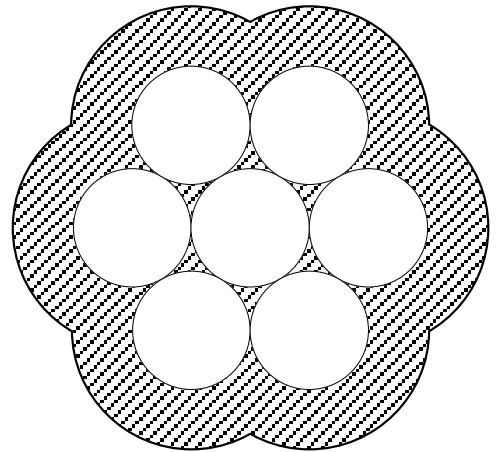

(6.5)

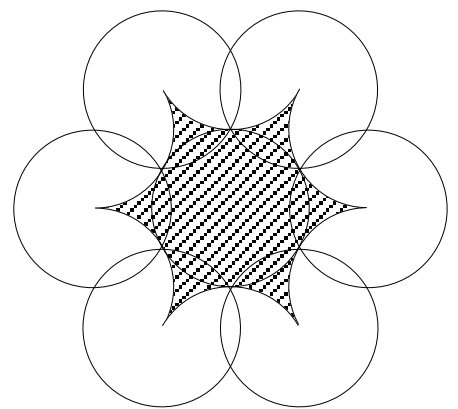

(6.8)

FIGURE 4. Economical clusters of unit circles

Except for some trivial cases, we do not know the solution to these problems even for $d=2$. However, some clusters of unit circles in $\mathbb{E}^{2}$, shown in Figure 4 , seem reasonably economical for the method described 
above. Using translates these clusters to estimate density bounds, we get the following:

$$
\begin{aligned}
\Delta_{2}\left(B^{2}\right) \geq \frac{3 \pi}{3 \sqrt{3}+8 \pi} & =0.31075 \ldots \\
\Delta_{3}\left(B^{2}\right) \geq \frac{3 \pi}{4 \sqrt{3}+6 \pi} & =0.36561 \ldots \\
\Delta_{7}\left(B^{2}\right) \geq \frac{7 \pi}{12 \sqrt{3}+8 \pi} & =0.47892 \ldots \\
\Theta_{3}\left(B^{2}\right) \leq \frac{6 \pi}{2 \sqrt{3}-\pi} & =58.44661 \ldots \\
\Theta_{4}\left(B^{2}\right) \leq \frac{4 \pi}{4-\pi} & =14.63916 \ldots \\
\Theta_{7}\left(B^{2}\right) \leq \frac{7 \pi}{6 \sqrt{3}-2 \pi} & =5.35179 \ldots .
\end{aligned}
$$

Clearly, these inequalities are far from sharp. Among good estimates for $\Delta_{n}\left(B^{2}\right)$ and $\Theta_{n}\left(B^{2}\right)$, one stands out. Clearly, any 1-saturated packing with unit balls becomes a covering if the radius of each ball is increased to 2. Since $\vartheta\left(B^{2}\right)=2 \pi / \sqrt{27}$ (a well-known result of Kershner [Ker39]), it follows that

$$
\Delta_{1}\left(B^{2}\right)=\pi / 6 \sqrt{3}=0.302299 \ldots
$$

Also, as we mentioned before,

$$
\Theta_{1}\left(B^{2}\right)=\Theta_{2}\left(B^{2}\right)=\infty .
$$

Apart from these three cases, it seems difficult to determine the exact values of $\Delta_{n}\left(B^{2}\right)$ and $\Theta_{n}\left(B^{2}\right)$.

\section{REMARKS, OPEN PROBLEMS AND CONJECTURES}

In relation to the conjecture stated in the introduction, claiming the existence of completely saturated packings and completely reduced coverings, observe the following:

(i): Complete saturation implies maximum density and complete reduction implies minimum density. More precisely, the density of a completely saturated packing with replicas of a body $K$ exists and is equal to $\delta(K)$. Similarly, the density of a completely reduced covering with replicas of $K$ exists and is $\vartheta(K)$.

(ii): Obviously, the converse of (i) is false. But a weaker statement holds: A periodic packing with replicas of $K$ with density $\delta(K)$ is completely saturated and a periodic covering with replicas of $K$ whose density is $\vartheta(K)$ is completely reduced.

The first observation indicates that the conjecture on existence of completely saturated packings and reduced coverings is not as obvious as it might appear. The conjecture, if true, would imply a version of Groemer's result [Gro63] on the existence of maximum density packings and minimum density coverings.

The second observation brings to mind the well-known problem: Given a body $K$, is there a periodic packing [covering] with replicas of $K$, whose density is $\delta(K)[\vartheta(K)]$ ? A positive answer to this question would imply our conjecture. However, Schmitt [Sch88] constructed a strictly star-shaped prototile for a monohedral tiling in $\mathbb{E}^{3}$ such that no tiling with its replicas is periodic, and by a slight modification of Schmitt's construction Conway produced a convex prototile with this property. For $\mathbb{E}^{2}$ no such example is known, but according to another result of Schmitt [Sch91] there is a strictly star-shaped set $K \subset \mathbb{E}^{2}$ whose replicas do not admit a periodic packing of density $\delta(K)$.

Generally, it seems extremely difficult to determine whether a given convex body admits a periodic packing (covering) of maximum (minimum) density. In particular, the answer is not known for the $d$-dimensional 
ball ( $d \geq 3$ ). The case $d=2$ offers some answers, since it is known (see [Fej50, Fej72]) that every centrallysymmetric convex disk attains its packing density in a lattice packing. The analogous statement for coverings is only a conjecture, supported by a partial result under the restriction to crossing-free coverings (see [Fej50, Fej72]).

There are only a handful of cases in which sphere packings in Euclidean or hyperbolic space are known to be completely saturated. Without exception, they follow from the Rogers [Rog58] and the Böröczky [Bör78] bounds: The density of any sphere packing in $d$ dimensions is at most the density in a regular simplex of $d+1$ kissing spheres with centers at the vertices of the simplex. If the regular simplex tiles space, there exists a corresponding periodic sphere packing that achieves the bound. The only regular simplices that tile Euclidean and hyperbolic space are:

•: Equilateral triangles in $\mathbb{E}^{2}$.

$\bullet:$ Triangles in $\mathbb{H}^{2}$ with angles of $2 \pi / n$ for $n \geq 7$.

•: Simplices in $\mathbb{H}^{4}$ with dihedral angles of $2 \pi / 5$.

The analogous bound for coverings also holds in Euclidean space (see Coxeter-Few-Rogers [CFR59]), although it is open for sphere coverings of hyperbolic $d$-space and the $d$-sphere for $d>2$. (For $\mathbb{H}^{2}$ the bound for circle coverings is due to L. Fejes Tóth [Fej64].) Therefore the same simplices also produce completely reduced coverings of the same types, except perhaps in $\mathbb{H}^{4}$.

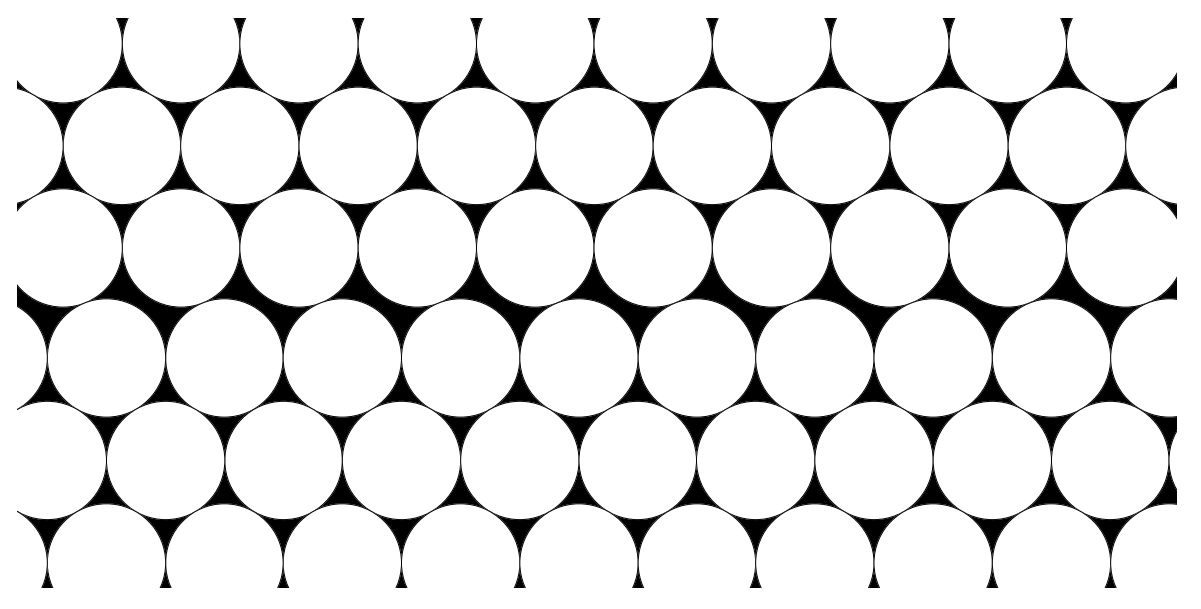

FIGURE 5. A sub-optimal packing which could be completely saturated

Clearly, the familiar densest lattice packing (covering) of $\mathbb{E}^{2}$ with unit circles is completely saturated (reduced), but we do not know whether there is a non-lattice, completely saturated packing (completely reduced covering) with unit circles. We do not even know whether or not the circle packing in Figure 5 is completely saturated. The arrangement of circles shown there is given by dividing lattice packing into two "half-plane" parts along a pair of adjacent rows of circles and then separating the parts slightly while maintaining contact between the two adjacent rows.

Although it seems difficult to determine $\Delta_{n}\left(B^{2}\right)$ and $\Theta_{n}\left(B^{2}\right)$, we conjecture that

$$
\Delta_{2}\left(B^{2}\right)=\pi(3-\sqrt{5}) / \sqrt{27}=0.461873 \ldots,
$$

which is the density of the packing shown in Figure 6. But we do not even have a conjecture for the other constants.

Theorem 5.1 relates the Newton covering number $N_{c}(K)$ for a convex body $K$ to $l(K)$. Newton covering numbers are of interest in their own right: Among all convex bodies in $d$ dimensions, which one has the 


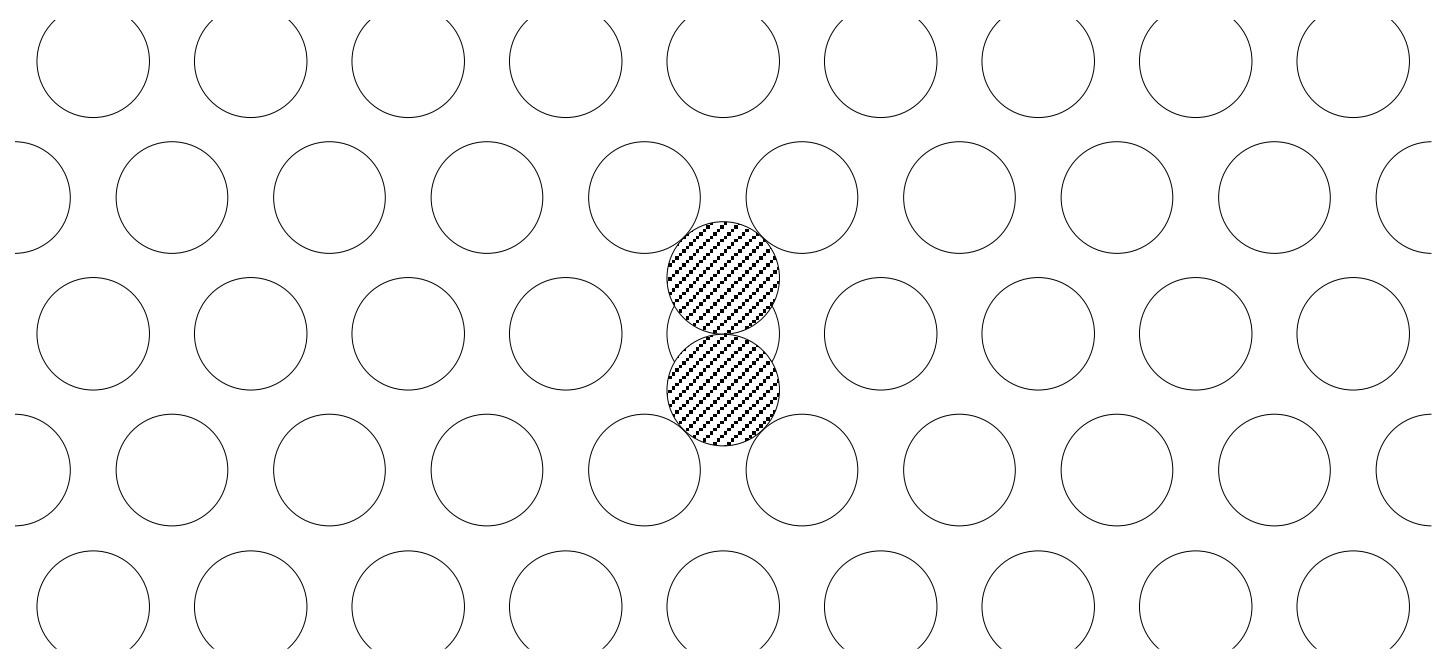

FIGURE 6. Possibly the least dense 2-saturated packing

greatest Newton covering number, and what is that number? Let $P$ denote the right pyramid over a $d-1$ dimensional cube $Q^{d-1}$, as in the remark following Theorem 5.8. Is $N_{c}(P)=N_{c}\left(Q^{d-1}\right)+1$ ? Does $N_{c}(P)$ depend on the height of the pyramid? What is the Newton covering number of the cube $Q^{d}$ ?

The inequality $l\left(B^{d}\right) \leq d+1$ (Theorem 5.6) is sharp for $d=2$ (see Figure 2), but we suspect that for $d \geq 3$ this is not the case. It might even turn out that $l\left(B^{d}\right)=3$ for all $d \geq 2$. This problem can be stated more simply as follows: Given a very dense covering of $\mathbb{E}^{d}$ with unit balls, can one always make a new covering by replacing three balls by two?

\section{REFERENCES}

[Bör74] K. Böröczky. Sphere packing in spaces of constant curvature I. Mat. Lapok, 25:265-306, 1974.

[Bör78] K. Böröczky. Packing of spheres in spaces of constant curvature. Acta Math. Acad. Sci. Hungar., 32:243-261, 1978.

[Bár87] I. Bárány. An extension of the Erdôs-Szekeres theorem on large angles. Combinatorica, 7:161-169, 1987.

[Bor63] A. Borel. Compact Clifford-Klein forms of symmetric spaces. Topology, 2:111-122, 1963.

[BP92] R. Benedetti and C. Petronio. Lectures on Hyperbolic Geometry. Springer-Verlag, Berlin-Heidelberg-New York, 1992.

[CFR59] H.S.M. Coxeter, L. Few and C.A. Rogers. Covering space with equal spheres. Mathematika, 6:147-157, 1959.

[ES61] P. Erdős and G. Szekeres. On some extremum problems in elementary geometry. Ann. Univ. Sci. Budapest, Eötvös Sect. Math., 3-4:53-62, 1960-61.

[Fej50] L. Fejes Tóth. Some packing and covering theorems. Acta Sci. Math. Szeged, 12/A:62-67, 1950.

[Fej64] L. Fejes Tóth. Regular Figures. Pergamon Press, Oxford, 1964.

[Fej72] L. Fejes Tóth. Lagerungen in der Ebene, auf der Kugel und im Raum. Springer-Verlag, New York-Berlin-Heidelberg, 2nd edition, 1972.

[FH80] L. Fejes Tóth and A. Heppes. Multi-saturated packings of circles. Studia Sci. Math. Hungar., 15:303-307, 1980.

[FK93] G. Fejes Tóth and W. Kuperberg. Packing and covering with convex sets. In P.M. Gruber and J.M. Wills, editors, Handbook of Convex Geometry, pages 799-860. North-Holland, Amsterdam-London-New York-Tokyo, 1993.

[Gro63] H. Groemer. Existensätze für Lagerungen im Euklidischen Raum. Math. Zitschr., 81:260-278, 1963.

[Had57] H. Hadwiger. Ungelöstes Problem Nr. 20. Elem. Math., 12:121, 1957.

[Ker39] R. Kershner. The number of circles covering a set. Amer. J. Math., 61:665-671, 1939.

[Mal40] A. I. Mal'cev. On the faithful representation of infinite groups by matrices. Mat. Sb., 8:405-422, 1940.

[Rog57] C.A. Rogers. A note on coverings. Mathematika, 4:1-6, 1957.

[Rog58] C.A. Rogers. The packing of equal spheres. Proc. London Math. Soc. (3), 8:609-620, 1958.

[RS57] C.A. Rogers and G.C. Shephard. The difference body of a convex body. Arch. Math., 8:220-233, 1957.

[Sch88] P. Schmitt. An aperiodic prototile in space. preprint, 1988.

[Sch91] P. Schmitt. Discs with special properties of densest packings. Discrete Comput. Geom., 6:181-190, 1991.

[Spa66] E.H. Spanier. Algebraic Topology. Springer-Verlag, New York-Berlin-Heidelberg, 1966. 
Gabor Fejes Tóth: Mathematical Institute of the Hungarian ACAdemy of Sciences, P.O.BOX 127, BUDAPEST, H-1364, HUNGARY.

E-mail address: gfejes@math-inst.hu

Greg Kuperberg: Department of Mathematics, Yale University, New Haven, CT 06520, U.S.A.

E-mail address: greg@math.yale.edu

Włodzimierz Kuperberg: DePartment of Mathematics, Auburn University, AL 36849-5310, U.S.A.

E-mail address: kuperwl@mail.auburn.edu 